\title{
OSCILLATIONS IN A NONAUTONOMOUS DELAY LOGISTIC DIFFERENCE EQUATION
}

\author{
by $\mathrm{CH}$. G. PHILOS
}

(Received 4th April 1990)

Consider the nonautonomous delay logistic difference equation

$$
\Delta y_{n}=p_{n} y_{n}\left(1-\frac{y_{n-t_{n}}}{K}\right),
$$

where $\left(p_{n}\right)_{n \geq 0}$ is a sequence of nonnegative numbers, $\left(l_{n}\right)_{n \geq 0}$ is a sequence of positive integers with $\lim _{n \rightarrow \infty}$ $\left(n-l_{n}\right)=\infty$, and $K$ is a positive constant. Only solutions which are positive for $n \geqq 0$ are considered. We established a sharp condition under which all solutions of $\left(E_{0}\right)$ are oscillatory about the equilibrium point $K$. Also we obtained sufficient conditions for the existence of a solution of $\left(E_{0}\right)$ which is nonoscillatory about $K$.

1980 Mathematics subject classification (1985 Revision). Primary 39A10.

\section{Introduction}

Difference equations provide an important first step in developing techniques for the analysis of dynamic phenomena in biology, both with respect to problem formulation and theoretical development. See, for example, $[1,4,5,7$ and 12]. An example is the logistic difference equation, which arises in models of population dynamics as a discretetime version of the logistic differential equation and as an approximation to a nonlinear renewal equation (see, e.g., $[3,4,7$ and 12]). This equation has been studied in many papers, including $[3,8,9,10$ and 13]. Our aim in this paper is to study the oscillation about the positive equilibrium point of the solutions of a nonautonomous delay logistic difference equation.

The logistic difference equation is

$$
N_{n+1}=N_{n}\left(a-b N_{n}\right)
$$

where $a$ and $b$ are positive constants. By writing

$$
y_{n}=\frac{b}{a} N_{n}
$$

the equation may be brought into canonical form 


$$
y_{n+1}=a y_{n}\left(1-y_{n}\right)
$$

This equation has two equilibrium points, the origin and $K=(a-1) / a$ which has physical meaning only for $a>1$. For $a>1$, the last equation takes the form

$$
\Delta y_{n}=p y_{n}\left(1-\frac{y_{n}}{K}\right)
$$

where $p=a-1>0$ and the forward difference operator $\Delta$ is defined as usual, i.e. $\Delta y_{n}=y_{n+1}-y_{n}$.

In this paper, we consider the nonautonomous delay logistic difference equation

$$
\Delta y_{n}=p_{n} y_{n}\left(1-\frac{y_{n-l_{n}}}{K}\right)
$$

where $\left(p_{n}\right)_{n \geqq 0}$ is a sequence of nonnegative numbers, $\left(l_{n}\right)_{n \geqq 0}$ is a sequence of positive integers with

$$
\lim _{n \rightarrow \infty}\left(n-l_{n}\right)=\infty
$$

and $K$ is a positive constant.

Throughout the paper we will use the notation

$$
l=-\min _{n \geqq 0}\left(n-l_{n}\right) .
$$

Clearly, $l$ is a positive integer.

By a solution of $\left(E_{0}\right)$ we mean a sequence $\left(y_{n}\right)_{n \geqq-l}$ which satisfies $\left(E_{0}\right)$ for all $n \geqq 0$.

Motivated by the plausible applications of $\left(E_{0}\right)$, in what follows we consider only solutions $\left(y_{n}\right)_{n \geqq-l}$ of $\left(E_{0}\right)$ with $y_{n}>0$ for every $n \geqq 0$.

We are concerned with the oscillation of the solutions of $\left(E_{0}\right)$ about the equilibrium point $K$. A solution $\left(y_{n}\right)_{n \geqq-l}$ of $\left(E_{0}\right)$ is said to be oscillatory about $K$ if the terms of the sequence are neither eventually greater than $K$ nor eventually less than $K$. Otherwise, the solution is called nonoscillatory about $K$.

The substitution

$$
x_{n}=\frac{y_{n}}{K}-1, n \geqq-l
$$

transforms $\left(E_{0}\right)$ into the equation

$$
\Delta x_{n}+p_{n}\left(1+x_{n}\right) x_{n-l_{n}}=0
$$


Clearly, the oscillation of $\left(y_{n}\right)_{n \geqq-1}$ about $K$ is equivalent with the usual oscillation (i.e. the oscillation about the origin) of $\left(x_{n}\right)_{n \geqq-1}$. As usual, a solution $\left(x_{n}\right)_{n \geqq-1}$ of $(E)$ is called oscillatory if it is neither eventually positive nor eventually negative, and otherwise the solution is called nonoscillatory.

Moreover, the fact that $y_{n}>0$ for $n \geqq 0$ is equivalent with the statement $1+x_{n}>0$ for $n \geqq 0$. So, in the sequel we consider only such solutions $\left(x_{n}\right)_{n \geqq-l}$ of $(E)$ which satisfy

$$
1+x_{n}>0 \text { for all } n \geqq 0 \text {. }
$$

The paper is organized as follows: In Section 2 we give a sharp condition for the oscillation of all solutions of $(E)$. Sufficient conditions for the existence of a nonoscillatory solution of $(E)$ are presented in Section 3.

For related results concerning the oscillation of the solutions of $(E)$ we refer to the recent paper [2]. Moreover, we note that oscillation and nonoscillation criteria for a nonautonomous delay logistic differential equation are obtained in [14].

\section{A sharp condition for oscillations}

The main result in this section is Theorem 1 below, which provides a sufficient condition for the oscillation of all solutions of the delay difference equation $(E)$ where the sequence $\left(n-l_{n}\right)_{n \geqq 0}$ is assumed to be increasing.

Theorem 1. Let the sequence $\left(n-l_{n}\right)_{n \geqq 0}$ be increasing and assume that

$$
\liminf _{n \rightarrow \infty}\left(\frac{1}{l_{n}} \sum_{k=n-l_{n}}^{n-1} p_{k}\right)>\limsup _{n \rightarrow \infty} \frac{l_{n}^{l_{n}}}{\left(l_{n}+1\right)^{l_{n}+1}}
$$

Then all solutions of $(E)$ are oscillatory.

In order to prove Theorem 1 we make use of the following known result, in which $\left(P_{n}\right)_{n \geqq 0}$ is assumed to be a sequence of nonnegative numbers.

Theorem 0. Let the sequence $\left(n-l_{n}\right)_{n \geqq 0}$ be increasing and assume that

$$
\liminf _{n \rightarrow \infty}\left(\frac{1}{l_{n}} \sum_{k=n-l_{n}}^{n-1} P_{k}\right)>\limsup _{n \rightarrow \infty} \frac{l_{n}^{l_{n}}}{\left(l_{n}+1\right)^{l_{n}+1}}
$$

Then:

(a) There is no sequence of numbers $\left(x_{n}\right)_{n \geqq-1}$ which is eventually positive and satisfies

$$
\Delta x_{n}+P_{n} x_{n-l_{n}} \leqq 0 \text { for all large } n \text {. }
$$

(b) There is no sequence of numbers $\left(x_{n}\right)_{n \geqq-1}$ which is eventually negative and satisfies 


$$
\Delta x_{n}+P_{n} x_{n-l_{n}} \geqq 0 \text { for all large } n \text {. }
$$

It has been proved in [11] (see also [6] for the special case where $l_{n}=l$ for all $n \geqq 0$ ) that, under the assumptions of Theorem 0 , all solutions of the linear delay difference equation

$$
\Delta x_{n}+P_{n} x_{n-l_{n}}=0
$$

are oscillatory. A slight modification in the proof of this result leads to Theorem 0 .

To prove Theorem 1 we also need the next lemma.

Lemma 1. Assume that

$$
\sum_{n=0}^{\infty} p_{n}=\infty
$$

Then every nonoscillatory solution of $(E)$ tends to zero as $n \rightarrow \infty$.

Proof. Let $\left(x_{n}\right)_{n \geqq-l}$ be a nonoscillatory solution of $(E)$. Then there exists an integer $n_{0} \geqq-l$ such that $\left(x_{n}\right)_{n \geqq n_{0}}$ is either positive or negative.

Consider first the case where $x_{n}>0$ for all $n \geqq n_{0}$. We choose a nonnegative integer $n_{1} \geqq n_{0}$ such that

$$
n-l_{n} \geqq n_{0} \quad \text { for every } n \geqq n_{1} \text {. }
$$

Then from $(E)$ it follows that $\Delta x_{n} \leqq 0$ for $n \geqq n_{1}$ and so the sequence $\left(x_{n}\right)_{n \geqq n_{1}}$ is decreasing. Hence, $\alpha \equiv \lim _{n \rightarrow \infty} x_{n}$ exists and is a nonnegative number. Assume, for the sake of contradiction, that $\alpha>0$. Then from $(E)$ we obtain

$$
\Delta x_{n}=-p_{n}\left(1+x_{n}\right) x_{n-l_{n}} \leqq-p_{n} x_{n-l_{n}} \leqq-\alpha p_{n}
$$

for all $n \geqq n_{2}$, where the integer $n_{2} \geqq n_{1}$ is chosen so that

$$
n-l_{n} \geqq n_{1} \quad \text { for every } n \geqq n_{2} \text {. }
$$

Hence, we get for $n \geqq n_{2}$

$$
x_{n+1}-x_{n_{2}}=\sum_{k=n_{2}}^{n} \Delta x_{k} \leqq-\alpha \sum_{k=n_{2}}^{n} p_{k}
$$

and consequently

$$
\alpha-x_{n_{2}} \leqq-\alpha \sum_{k=n_{2}}^{\infty} p_{k},
$$


which contradicts (ii). Thus, $\alpha \equiv \lim _{n \rightarrow \infty} x_{n}$ must be zero.

Suppose now that $x_{n}<0$ for all $n \geqq n_{0}$ and choose the nonnegative integer $n_{1} \geqq n_{0}$ as above. By our general hypothesis, we have $1+x_{n}>0$ for $n \geqq 0$. From $(E)$ we derive $\Delta x_{n} \geqq 0$ for every $n \geqq n_{1}$, which means that $\left(x_{n}\right)_{n \geqq n_{1}}$ is an increasing sequence. This implies that $\beta \equiv \lim _{n \rightarrow \infty} x_{n}$ exists and is a nonpositive number. Let $\beta$ be negative. Then from $(E)$ it follows that

$$
\Delta x_{n}=-p_{n}\left(1+x_{n}\right) x_{n-l_{n}} \geqq-\left(1+x_{n_{2}}\right) \beta p_{n}
$$

for every $n \geqq n_{2}$, where the integer $n_{2} \geqq n_{1}$ is chosen as in the first case. So, we obtain for $n \geqq n_{2}$

$$
x_{n+1}-x_{n_{2}}=\sum_{k=n_{2}}^{n} \Delta x_{k} \geqq-\left(1+x_{n_{2}}\right) \beta \sum_{k=n_{2}}^{n} p_{k}
$$

and hence

$$
\beta-x_{n_{2}} \geqq-\left(1+x_{n_{2}}\right) \beta \sum_{k=n_{2}}^{\infty} p_{k},
$$

where $1+x_{n_{2}}>0$. This contradicts (ii) and so $\beta \equiv \lim _{n \rightarrow \infty} x_{n}=0$.

The proof of the lemma is complete.

Proof of Theorem 1. Assume, for the sake of contradiction, that there is a nonoscillatory solution $\left(x_{n}\right)_{n \geqq-l}$ of the difference equation $(E)$. Then we can choose an integer $n_{0} \geqq-l$ so that the sequence $\left(x_{n}\right)_{n \geqq n_{0}}$ is either positive or negative. We consider the following two cases:

Case I: $x_{n}>0$ for all $n \geqq n_{0}$. Let $n_{1} \geqq n_{0}$ be a nonnegative integer such that

$$
n-l_{n} \geqq n_{0} \quad \text { for every } n \geqq n_{1} \text {. }
$$

Then from $(E)$ it follows that

$$
\Delta x_{n}+p_{n} x_{n-l_{n}} \leqq 0 \text { for all } n \geqq n_{1}
$$

By Theorem 0, this is a contradiction.

Case 2: $x_{n}<0$ for all $n \geqq n_{0}$. In view of condition (i), we can choose a number $\varepsilon \in(0,1)$ such that

$$
(1-\varepsilon) \liminf _{n \rightarrow \infty}\left(\frac{1}{l_{n}} \sum_{k=n-l_{n}}^{n-1} p_{k}\right)>\limsup _{n \rightarrow \infty} \frac{l_{n}^{l_{n}}}{\left(l_{n}+1\right)^{l_{n}+1}}
$$


That is

$$
\liminf _{n \rightarrow \infty}\left(\frac{1}{l_{n}} \sum_{k=n-l_{n}}^{n-1} P_{k}\right)>\limsup _{n \rightarrow \infty} \frac{l_{n}^{l_{n}}}{\left(l_{n}+1\right)^{l_{n}+1}}
$$

where

$$
P_{n}=(1-\varepsilon) p_{n} \geqq 0 \quad(n=0,1, \ldots) .
$$

Furthermore, we observe that from condition (i) it follows that

$$
\liminf _{n \rightarrow \infty} \sum_{k=n-l_{n}}^{n-1} p_{k}>0
$$

which obviously implies that condition (ii) is satisfied. Hence, in view of Lemma 1 , we have

$$
\lim _{n \rightarrow \infty} x_{n}=0
$$

Thus, if we consider a nonnegative integer $n_{1}^{*} \geqq n_{0}$ such that

$$
n-l_{n} \geqq n_{0} \quad \text { for } \quad n \geqq n_{1}^{*}
$$

and

$$
x_{n}>-\varepsilon \text { for all } n \geqq n_{1}^{*},
$$

then from $(E)$ we obtain for $n \geqq n_{1}^{*}$

$$
0=\Delta x_{n}+p_{n}\left(1+x_{n}\right) x_{n-l_{n}} \leqq \Delta x_{n}+p_{n}(1-\varepsilon) x_{n-l_{n}} .
$$

So, we have

$$
\Delta x_{n}+P_{n} x_{n-l_{n}} \geqq 0 \text { for all } n \geqq n_{1}^{*}
$$

In view of Theorem 0 , this is impossible.

The proof of the theorem is now complete.

\section{Sufficient conditions for the existence of nonoscillatory solutions}

The main purpose in this section is to prove the following theorem, which establishes 
some conditions under which the delay difference equation $(E)$ has a nonoscillatory solution.

Theorem 2. Suppose that

$$
\sum_{i=0}^{L_{n}-1} p_{n+i}>0 \quad \text { for all } n \geqq 0
$$

where

$$
L_{n}=\max \left\{\rho \in\{1,2, \ldots\}:-l \leqq \mu-l_{\mu} \leqq n-1 \quad \text { for } \quad n \leqq \mu \leqq n+\rho-1\right\} \quad(n=0,1, \ldots) .
$$

Moreover, assume that there exist two numbers $c$ and $\gamma$ with $0<c<\gamma<1$ such that

$$
p_{n}<\gamma \quad \text { for every } n \geqq 0
$$

and

$$
\frac{1}{\gamma} \prod_{k=n-l_{n}}^{n-1}\left(1-\frac{1}{\gamma} \tilde{p}_{k}\right) \geqq 1+\left(1-\frac{1}{\gamma} c\right)^{l+1} \text { for all } n \geqq 0
$$

where

$$
\tilde{p}_{n}=\left\{\begin{array}{l}
p_{n}, \text { if } \quad n \geqq 0 \\
c, \text { if } \quad-l-1 \leqq n<0 .
\end{array}\right.
$$

Then there exists a positive solution $\left(x_{n}\right)_{n \geqq-1}$ of $(E)$ with $\lim _{n \rightarrow \infty} x_{n}=0$.

We remark that in the special case where $l_{n}=l(n=0,1, \ldots)$ we also have $L_{n}=l$ for all $n \geqq 0$. The proof of Theorem 2 is based on the following lemma.

Lemma 2. Suppose that (iii) holds, where $\left(L_{n}\right)_{n \geqq 0}$ is defined as in Theorem 2 , and let $\left(z_{n}\right)_{n \geqq-l}$ be a positive solution of the delay difference inequality

$$
\Delta z_{n}+p_{n}\left(1+z_{n}\right) z_{n-l_{n}} \leqq 0
$$

Then there exists a positive solution $\left(x_{n}\right)_{n \geqq-1}$ of $(E)$ with $\lim _{n \rightarrow \infty} x_{n}=0$ and such that $x_{n} \leqq z_{n}$ for $n \geqq-l$.

Note. By a solution of (I) we mean a sequence $\left(z_{n}\right)_{n \geqq-l}$ which satisfies (I) for all $n \geqq 0$.

Proof. The method of proof is similar to that of a lemma in [11] (see also the proof of Theorem 3 in [6] for the special case where $l_{n}=l$ for $\left.n=0,1, \ldots\right)$.

From (I) it follows that for $v \geqq n \geqq 0$ 


$$
z_{n}>-\left(z_{v+1}-z_{n}\right)=-\sum_{k=n}^{v} \Delta z_{k} \geqq \sum_{k=n}^{v} p_{k}\left(1+z_{k}\right) z_{k-l_{k}}
$$

and consequently

$$
z_{n} \geqq \sum_{k=n}^{\infty} p_{k}\left(1+z_{k}\right) z_{k-l_{k}} \quad \text { for every } \quad n \geqq 0
$$

Next, we consider the space $\mathscr{X}$ of all sequences $\left(x_{n}\right)_{n \geqq-l}$ which satisfy

$$
x_{n}=z_{n} \text { for }-l \leqq n<0, \text { and } 0 \leqq x_{n} \leqq z_{n} \text { for } n \geqq 0
$$

For any sequence $\left(x_{n}\right)_{n \geqq-l}$ in $\mathscr{X}$, we put

$$
S x_{n}=\left\{\begin{array}{l}
z_{n}, \quad \text { if } \quad-l \leqq n<0 \\
\sum_{k=n}^{\infty} p_{k}\left(1+x_{k}\right) x_{k-l_{k}}, \quad \text { if } \quad n \geqq 0
\end{array}\right.
$$

We immediately see that this formula defines an operator $S: \mathscr{X} \rightarrow \mathscr{X}$. This operator is monotonic in the sense that, if $\left(x_{n}^{1}\right)_{n \geqq-l}$ and $\left(x_{n}^{2}\right)_{n \geqq-l}$ are two sequences in $\mathscr{X}$ with $x_{n}^{1} \leqq x_{n}^{2}$ for $n \geqq-l$, then $S x_{n}^{1} \leqq S x_{n}^{2}$ for all $n \geqq-l$. Now, we define

$$
x_{n}^{0}=z_{n} \text { for } n \geqq-l
$$

and

$$
x_{n}^{r}=S x_{n}^{r-1} \text { for } n \geqq-l \quad(r=1,2, \ldots)
$$

and we see that $\left(x_{n}^{r}\right)_{n \geqq-l}$ belongs to $\mathscr{X}$ for every nonnegative integer $r$. Moreover, it is easy to see that

$$
x_{n}^{0} \geqq x_{n}^{1} \geqq x_{n}^{2} \geqq \cdots \quad \text { for all } n \geqq-l \text {. }
$$

Furthermore, we set

$$
x_{n}=\lim _{r \rightarrow \infty} x_{n}^{r} \text { for } n \geqq-l
$$

and we observe that $0 \leqq x_{n} \leqq z_{n}$ for every $n \geqq-l$. Moreover, we have $x_{n}=S x_{n}$ for all $n \geqq-l$ and consequently 


$$
x_{n}=\left\{\begin{array}{l}
z_{n}, \quad \text { if }-l \leqq n<0 \\
\sum_{k=n}^{\infty} p_{k}\left(1+x_{k}\right) x_{k-l_{k}}, \text { if } n \geqq 0
\end{array}\right.
$$

This gives

$$
\lim _{n \rightarrow \infty} x_{n}=0
$$

Also, we get

$$
\Delta x_{n}=-p_{n}\left(1+x_{n}\right) x_{n-l_{n}} \text { for all } n \geqq 0
$$

and therefore the sequence $\left(x_{n}\right)_{n \geqq-l}$ is a solution of $(E)$. Since $x_{n}=z_{n}>0$ for $-l \leqq n<0$, it remains to show that $x_{n}>0$ for all $n \geqq 0$. Assume, for the sake of contradiction, that the sequence $\left(x_{n}\right)_{n \geqq 0}$ has at least one zero. Then these exists an integer $n_{0} \geqq 0$ such that

$$
x_{n}>0 \text { for }-l \leqq n<n_{0} \text {, and } x_{n_{0}}=0 \text {. }
$$

By condition (iii) and the definition of the integer $L_{n_{0}}$, we obtain

$$
\begin{aligned}
0 \leqq & x_{n_{0}}+L_{n_{0}}=x_{n_{0}+L_{n 0}}-x_{n_{0}}=\sum_{\mu=n_{0}}^{n_{0}+L_{n_{0}}-1} \Delta x_{\mu} \\
& =-\sum_{\mu=n_{0}}^{n_{0}+L_{n_{0}}-1} p_{\mu}\left(1+x_{\mu}\right) x_{\mu-l_{\mu}} \\
& \leqq-\left[\min _{n_{0} \leqq \mu \leqq n_{0}+L_{n_{0}}-1}\left(1+x_{\mu}\right) x_{\mu-l_{\mu}}\right] \sum_{\mu=n_{0}}^{n_{0}+L_{n_{0}}-1} p_{\mu} \\
& =-\left[\min _{n_{0} \leqq \mu \leqq n_{0}+L_{n_{0}}-1}\left(1+x_{\mu}\right) x_{\mu-l_{\mu}}\right] \sum_{i=0}^{L_{n_{0}}-1} p_{n_{0}+i} \\
& <0,
\end{aligned}
$$

which is a contradiction, and so the proof of our lemma is complete.

Proof of Theorem 2. Define (cf. [11, Theorem 5] or [6, Corollary 1]) 


$$
z_{n}=\prod_{k=-l-1}^{n-1}\left(1-\frac{1}{\gamma} \tilde{p}_{k}\right) \text { for } n \geqq-l
$$

By taking into account the fact that $0<c<\gamma$ and condition (iv), we can see that $z_{n}>0$ for all $n \geqq-l$. Furthermore, by using condition (v), we derive for each $n \geqq 0$

$$
\begin{aligned}
\Delta z_{n} & =\left[\left(1-\frac{1}{\gamma} \tilde{p}_{n}\right)-1\right] \prod_{k=-l-1}^{n-1}\left(1-\frac{1}{\gamma} \tilde{p}_{k}\right) \\
& =-\frac{1}{\gamma} p_{n}\left[\prod_{k=-l-1}^{n-l_{n}-1}\left(1-\frac{1}{\gamma} \tilde{p}_{k}\right)\right]\left[\prod_{k=n-l_{n}}^{n-1}\left(1-\frac{1}{\gamma} \tilde{p}_{k}\right)\right] \\
& =-p_{n} z_{n-i_{n}}\left[\frac{1}{\gamma} \prod_{k=n-l_{n}}^{n-1}\left(1-\frac{1}{\gamma} \tilde{p}_{k}\right)\right] \leqq-p_{n} z_{n-l_{n}}\left[1+\left(1-\frac{1}{\gamma} c\right)^{l+1}\right] \\
& \leqq-p_{n} z_{n-l_{n}}\left[1+\prod_{k=-l-1}^{n-1}\left(1-\frac{1}{\gamma} \tilde{p}_{k}\right)\right]=-p_{n} z_{n-l_{n}}\left(1+z_{n}\right) .
\end{aligned}
$$

Hence, the sequence $\left(z_{n}\right)_{n \geqq-l}$ is a positive solution of the delay difference inequality (I). So, an application of Lemma 2 completes our proof.

\section{REFERENCES}

1. K. L. Cooke, D. F. Calef and E. V. Level, Stability or chaos in discrete epidemic models, in: Nonlinear Systems and Applications: An International Conference (Edited by V. Lakshmikantham, Academic Press, New York, 1977), 73-93.

2. L. H. Erbe and B. G. Zhang, Oscillation of discrete analogues of delay equations, Differential and Integral Equations 2 (1989), 300-309.

3. F. C. Hoppensteadt and J. M. Hyman, Periodic solutions of a logistic difference equation, SIAM J. Appl. Math. 32 (1977), 73-81.

4. J. N. KaPUR, Mathematical Modelling (John Wiley and Sons, New York, 1988).

5. G. LADAs, Recent developments in the oscillation of delay difference equations (International Conference on Differential Equations: Theory and Applications in Stability and Control, Colorado Springs, Colorado, June 7-10, 1989).

6. G. Ladas, $\mathrm{C}_{\mathrm{H}}$. G. Philos and Y. G. Sficas, Sharp conditions for the oscillation of delay difference equations, J. Appl. Math. Simulation 2 (1989), 101-111.

7. V. Lakshmikantham and D. Trigiante, Theory of Difference Equations: Numerical Methods and Applications (Academic Press, New York, 1988).

8. T. Y. LI and J. A. Yorke, Period three implies chaos, Amer. Math. Monthly 82 (1975), 985-992. 
9. R. M. MAY, Biological populations with nonoverlapping generations; stable points, stable cycles, and chaos, Science 186 (1974), 645-647.

10. R. M. MAY, Simple mathematical models with very complicated dynamics, Nature 261 (1976), 459-467.

11. Сн. G. Philos, On oscillations of some difference equations, Funkcial. Ekvac., to appear.

12. M. R. Rose, Quantitative Ecological Theory: An Introduction to Basic Models (Croom Helm, London, 1987).

13. Guo Ben-Yu, B. D. Sleeman and Chen Sui-Yang, On the discrete logistic model of biology, Appl. Anal. 33 (1989), 215-231.

14. B. G. Zhang and $K$. Gopalsamy, Oscillation and nonoscillation in a nonautonomous delay-logistic equation, Quart. Appl. Math. 46 (1988), 267-273.

Department of Mathematics

UNIVERSITY OF IOANNINA

P.O. Box 1186

45110 Ioannina, Greece 